\title{
Breathing lung transplantation with the Organ Care System (OCS) Lung: lessons learned and future implications
}

\author{
William Lightle, Daoud Daoud, Gabriel Loor \\ Michael E. DeBakey Department of Surgery, Baylor College of Medicine, Houston, TX, USA \\ Contributions: (I) Conception and design: All authors; (II) Administrative support: All authors; (III) Provision of study materials or patients: All \\ authors; (IV) Collection and assembly of data: All authors; (V) Data analysis and interpretation: All authors; (VI) Manuscript writing: All authors; (VII) \\ Final approval of manuscript: All authors. \\ Correspondence to: Gabriel Loor, MD. Baylor College of Medicine, Baylor St. Luke's Medical Center, 6770 Bertner Avenue, Suite C355, Houston, TX \\ 77030, USA. Email: Gabriel.Loor@bcm.edu.
}

\begin{abstract}
Ex vivo lung perfusion (EVLP) represents a potentially important advancement in the preservation of donor lungs prior to transplantation. Portable EVLP or "Breathing Lung Transplantation" with the Organ Care System (OCS) Lung combines the fundamental components of EVLP with portability, thus reducing the total ischemic burden. The Food and Drug Administration (FDA) approved OCS for perfusion of standard donor lungs prior to transplant in 2018. The current review discusses the available literature on the clinical outcomes of OCS Lung as well as translational data.
\end{abstract}

Keywords: Ex vivo lung perfusion (EVLP); lung transplantation; Organ Care System (OCS); donor lung

Submitted Feb 12, 2019. Accepted for publication Mar 12, 2019.

doi: $10.21037 /$ jtd.2019.03.32

View this article at: http://dx.doi.org/10.21037/jtd.2019.03.32

\section{End stage lung disease}

Chronic lung disease is the fourth leading cause of death in the United States and is a major health burden worldwide (1). Lung transplant is a treatment option for patients with cystic fibrosis, chronic obstructive pulmonary disease (COPD), interstitial lung disease, pulmonary hypertension and other chronic lung diseases. There are approximately 4,500 lung transplants performed annually world-wide and the number continues to grow (2). Yet there are significant limitations with transplant. One of the biggest challenges is the critical organ shortage. Patients have a $15-30 \%$ chance of dying on the waiting list depending on their diagnosis and urgency (3). Currently, only $20 \%$ of organ offers are transplanted. Thus, $80 \%$ of lungs are discarded. Another challenge is primary graft dysfunction (PGD) which is described below. Finally, the quality of the organ at the time of transplant can contribute to chronic lung allograft dysfunction (CLAD), which occurs in $50 \%$ of patients by five years after transplant.

\section{PGD}

Up to $30 \%$ of transplanted lungs will develop PGD. PGD is graded from zero to three, with three being the worst category of graft dysfunction. PGD is associated with a high rate of mortality in the hospital and following discharge (4).

An important donor risk factor for development of PGD is cigarette smoking. Other risk factors may include aspiration, chest trauma, lung contusions, undersized donors relative to recipient, and heavy alcohol use (5). Recipient risk factors for PGD include pre-transplant lung diseases other than COPD or cystic fibrosis, obesity and pulmonary hypertension. Importantly, prolonged cold ischemic time is associated with PGD.

\section{Ex vivo lung perfusion (EVLP)}

EVLP describes a concept of perfusing and ventilating a donor lung outside of the donor and recipient. Breathing lung transplantation is a form of EVLP. The Organ Care 


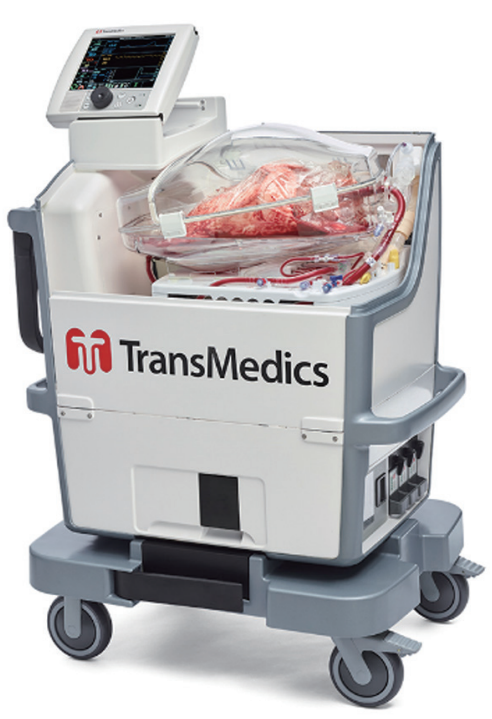

Figure 1 The OCS Lung is a portable EVLP device often described as "breathing lung transplantation." Warm blood flows through the lungs while the lung is ventilated. (Used with permission from TransMedics). OCS, Organ Care System; EVLP, ex vivo lung perfusion.

System (OCS) Lung (TransMedics, Andover, MA, USA) is currently the only device approved by the Food and Drug Administration for portable normothermic EVLP. EVLP platforms are either static or portable. Portable normothermic EVLP is often referred to as "breathing lung transplantation." In static EVLP, the perfusion device stays at the recipient institution or at a perfusion center. Portable EVLP relies on normothermic, blood-based perfusion, ventilation, monitoring and recruitment (6). Portable EVLP addresses the issue of cold ischemia by maintaining the organ in a physiologic state throughout transportation (7). Additives such as steroids, antibiotics, glucose, multivitamins and insulin aid this process.

Instrumentation for portable EVLP begins with a pulmonary artery cannula that connects to the pulmonary artery. Blood pumps through the lungs, and then drains passively through the left atrium. After flowing through the left atrium, the blood flows into a reservoir, and a pulsatile pump propels it through an oxygenator with a set temperature. Finally, the blood flows through the pulmonary artery where the cycle repeats (Figure 1).

The portable EVLP includes a catheter that helps monitor pulmonary artery pressures. It also includes a miniaturized ventilator to ventilate the lung. One can choose different settings to optimize the organ's preservation. A Blue-tooth enabled monitor allows the team to manipulate ventilator and flow settings and track the progress of the organ during travel.

There are several investigational studies in the literature regarding EVLP $(8,9)$. Yet there are a limited number of prospective clinical trials. Portable normothermic EVLP was first described by Warnecke and colleagues in a series of 12 patients (7). All patients were transplanted with lungs perfused on the OCS Lung system and all survived to discharge. Subsequently, the INSPIRE trial in 2018 became the largest randomized controlled multicenter trial in the history of lung preservation (10). The inclusion criteria were as follows: (I) standard criteria lung donors for bilateral lung transplantation; (II) pressure of oxygen in arterial blood $\left(\mathrm{PaO}_{2}\right)$ : fraction of inspired oxygen $\left(\mathrm{FiO}_{2}\right)>300 \mathrm{mmHg}$; (III) age $<65$ years; (IV) organ suitable for either cold storage or OCS Lung; (V) no active pulmonary disease and (VI) standard brain-dead organ donation. This trial randomized 370 patients to OCS lung or ice. The patient distribution consisted of 182 patients in the control group (ice), and 188 patients in the OCS group. The composite primary effectiveness endpoint was absence of PGD3 $\left(\mathrm{PaO}_{2} / \mathrm{FiO}_{2}\right.$ $<200$, and diffuse allograft infiltrates on chest radiograph) within 72 hours and survival at 30 days post-transplant.

This was one of the first large scale attempts to intervene on the donor and reduce PGD. It also provided a prospective assessment of the incidence of PGD after lung transplant to corroborate prior retrospective and single center experiences. The results showed a $30 \%$ chance of developing PGD3 with standard ice preservation, which was consistent with prior observations $(4,11)$. Of note, this trial used the ISHLT 2005 scoring system for PGD. There have since been some important modifications and caveats that may alter the perceived incidence of PGD3 within 72 hours (12). When the lungs came on OCS, there was a $50 \%$ less incidence of developing PGD3 within 72 hours. Interestingly, despite the decreased chance of developing PGD3, long-term survival was not statistically different. This suggests that the centers involved in the trials were well versed and capable of rescuing patients through this event. Comparing the number of days on the ventilator and length of hospital stay, the OCS group had a trend towards less days than the ice group, but this was not statistically significant. Overall, survival was the same and PGD was decreased.

Can we extrapolate these findings with standard donor lungs in the INSPIRE trial into extended criteria lung 
transplantation to increase donor utilization? The EXPAND I trial was designed as a large prospective multicenter international trial looking specifically at extended criteria donors (ECD). The objective was to evaluate the safety and efficacy of the OCS lung system to recruit, preserve and assess non-ideal or ECD lungs that fail to meet commonly accepted donor lung acceptance criteria for transplantation. This was a prospective single arm multi-center international pivotal trial focusing on improving utilization of nonideal donor lungs for double lung transplantation. The inclusion criteria were as follows: (I) donor $\mathrm{PaO}_{2} / \mathrm{FiO}_{2}$ $\leq 300$; (II) expected ischemic time $>6 \mathrm{~h}$; (III) donation after cardiac death (DCD) donors; and (IV) donor age $\geq 55$ years. Preliminary results of this trial were presented at the ISHLT in Nice, France in 2018 and the final manuscript is currently in preparation (13). This study involved 93 lungs that were perfused on OCS-12 of which did not meet criteria. This was different than the INSPIRE trial where recipients were already in the operating room by the time the device arrived in the hospital and the organs had already been expected to meet criteria for transplantation. In the EXPAND I trial; the surgeon waited to assess the organ and ensure that it met standard criteria after preservation.

According to the ISHLT presentation, 81 of the donor organs met inclusion criteria for transplant after preservation for a utilization rate of $87 \%$. This is the highest yield rate for ECD in a multicenter EVLP trial. Ultimately, 79 of those 81 lungs were transplanted. The EXPAND I trial reported an average total ischemic time of 10 hours. The majority of this time was spent in machine perfusion, while a minority was cold ischemic time and warm ischemic time for sewing the organ. Overall vascular resistance, peak airway pressures and blood gas ratios improved from start to finish in the organs that were transplanted. Following transplantation, the survival rate was $98 \%$ at 30 days. These results compared favorably to the standard ice group in INSPIRE and also to national benchmarks for survival.

Even after OCS, PGD3 rates were higher than the INSPIRE trial. This makes intuitive sense since these were ECD and until now, there have not been clear reports on the rate of PGD in such a cohort. There are only two additional multi-centered trials on EVLP and ECD to our knowledge. One has been completed (DEVELOP UK Trial which used a static system), and one is still in process (Novel trial). The DEVELOP UK Trial time 0 PGD3 rate was reported at $88 \%$ (14). At time 72 hours (T72), the PGD3 rate was $27 \%$. The rate of PGD3 at T72 for EXPAND was reported as $6 \%$ and time 0 was $44 \%$ as described at the ISHLT presentation. Thus, both of these studies showed higher PGD3 than the INSPIRE trial, but rates that decreased with time. It is also important to note that the grading of PGD is not consistent across studies, so it is not scientifically correct to compare PGD3 rates between studies. There are several caveats and revisions in the grading systems, as well as differences in the chest $\mathrm{x}$ ray interpretations that could lead to variation in results.

In the NOVEL trial, a static EVLP system was used to evaluate ECD including DCD. DCD lung transplants resulted in higher PGD scores than in non-DCD transplants (15). The same was true in the EXPAND trial. Both trials showed improved utilization rates compared to standard ice preservation. The preliminary data presented for the Novel trial showed a $51 \%$ utilization rate, and for the EXPAND trial, an $87 \%$ utilization rate $(13,15,16)$.

Schiavon and colleagues reported a smaller single center experience in Italy using 8 ECD perfused on the OCS Lung (17). In this pilot study, all 8 donors proceeded to transplant. Their inclusion criteria varied slightly from EXPAND by including donors with pulmonary edema. Their study showed improvements in physiology and oxygenation in the donor grafts. Utilization was 100\% and one patient had PGD3 at 72 hours. Median ventilation time was 60 hours, while ICU and hospital stay were 14 and 36 days respectively. In hospital mortality occurred in one patient due to pancreatitis and myocardial infarction.

Zeriouh and colleagues reported their findings with 322 consecutive lung transplants using OCS in 14 extended criteria cases (18). They compared their donor and recipient demographics and outcomes. There were greater number of heavy smokers (>20 pack year history) in the OCS group. There were 3 DCD cases in the OCS group compared with 6 in the cold storage group $(\mathrm{P}=0.741)$. There was a non-significant trend towards better PF ratios in the OCS group within 72 hours. The OCS group showed superior forced expiratory volume-one second (FEV1) values at 3- and 6-month post-transplant. There was no difference in overall survival or incidence of bronchiolitis obliterans syndrome (BOS) between groups. This is an important real-world experience that adds to the body of evidence supporting the safety of OCS in ECD.

In summary, portable EVLP is a safe way to extend ischemic times in standard donor lungs. It results in better preservation than ice for standard donor lungs as evidenced by the decreased incidence of PGD3 in the INSPIRE trial, but equivalent one to two-year survival. It has demonstrated excellent survival and utilization with ECD in 
the preliminary EXPAND data, but at the potential expense of higher PGD rates than that seen in standard donor lungs. Thus, there remains room to improve upon these advancements with a focus on organ repair and recovery.

\section{Future directions}

\section{Split lung ventilation}

There are a several efforts underway to optimize the results of portable EVLP. One of these includes split lung preservation. Split lung preservation allows the use of OCS for single lungs and reduces the amount of time the organ is not perfused with blood. One organ is removed from the module, while the other is left ventilated and perfused until it is ready for transplant. This could improve the results in the INSPIRE trial by shortening the cold ischemic time even further. Other advances include the ability to perform lobectomies and wedge resections on the device, which may allow resection of nodules or size reduction in order to further expand the donor pool.

\section{Blood perfusates for prolonged preservation}

Another topic of discussion on future advancements with EVLP includes the use of whole blood versus banked blood. Animal studies at the University of Minnesota demonstrated that autologous whole blood from the donor may provide greater stability than banked blood for longer preservation intervals. Longer preservation intervals are necessary when the organ requires further intervention to allow cellular repair and regeneration. During our animal lab experiments, the amount of edema noted over 24 hours with perfusion of whole blood was significantly less than the amount that was observed with banked blood (19). Moreover, our group at the University of Minnesota published a report on the feasibility of collecting this blood during multi-organ procurements (20).

Sommer and colleagues performed 24 left single lung porcine transplants after 24-hour perfusion on OCS using a variety of perfusates (21). They compared STEEN's solution enriched with erythrocytes, acellular STEEN's solution, and low-potassium dextran solution enriched with erythrocytes. They showed that physiologic variables such as peak airway pressure and pulmonary vascular resistance are reliable early and late predictors of transplant outcomes, respectively. STEEN solution with erythrocytes had superior survival and oxygenation post-transplant. This study along with the University of Minnesota experience suggests that there are several options for optimizing prolonged preservation.

A pilot study presented by the University of Minnesota group at ISHLT in 2018, showed that prolonged EVLP in human lungs rejected for transplant with autologous whole blood for 24 hours was safe and feasible (22). Ten ECD lungs that were not candidates for the EXPAND trial were tested on the device. Five of these lungs were deemed transplantable by two different surgeons due to their improved blood gas ratios, vascular resistance and pulmonary artery pressures. This study did not proceed to transplantation, as it was mostly assessing feasibility and safety. Interestingly, there were certain physiologic markers associated with the device early on (e.g., the amount of PF ratio and the amount of pulmonary vascular resistance) that predicted whether the organ would be transplantable or not after 24 hours.

\section{OCS Lung recovery after DCD injury—translational experience}

Mohite and colleagues reported the first case in the literature from Europe using the OCS Lung system for DCD lungs (23). Several potential injuries occur with DCD. The donor is not yet brain dead, but the family has allowed withdrawal of care. The breathing tube is removed and there is a variable period of agonal phase before the donor expires. After a short period of cardiac arrest, the donor team can procure the organs without circulating blood. Thus, the evaluation of the organ is limited and there is variable opportunity for ischemic injury during the DCD process. DCD is a highly underutilized resource and accounts for less than $6 \%$ of lung transplants in the United States. In the Mohite report, the lungs performed well on OCS and were transplanted successfully into a patient with COPD who did well and was discharged to home after 14 days.

Additional work by the lab at the University of Minnesota demonstrated the effects of OCS preservation after 1-2 hours of un-resuscitated cardiac arrest or warm ischemic time. This is akin to uncontrolled DCD scenarios whereby the arrest occurs prior to the procurement team's arrival. The lab showed that one hour of warm ischemia was well tolerated with results that were similar to controls as long as the lung was ventilated during that arrest period (24). Ice was not used during the downtime, which may have extended this interval even further. Moreover, a follow-up study transplanting the 
organs after resuscitation showed that survival was excellent even after 2 hours post cardiac arrest followed by 24 hours of OCS preservation (25). However, there was still room for improvement regarding oxygenation, which remained in the 200 range for the DCD lungs.

Interestingly, there were several predictors on OCS that revealed the function of the donor lung after transplant. For instance, elevated pulmonary artery pressures at 8 hours on OCS correlated with worse oxygen levels in the recipient. Increased organ weight throughout preservation was associated with worse outcomes. Increased vascular resistance was associated with worse PF ratios. The postEVLP blood gas ratios correlated with the post-transplant allograft $\mathrm{PF}$ ratios although this relation did not reach statistical significance. It is critical that we learn from our EVLP experiences and acquire a sense of confidence in biomarkers and physiologic profiles that predict a good outcome.

Sommer and colleagues utilized a 24-hour cold ischemic injury model to demonstrate the ability of OCS to recondition lungs after ischemia (21). As mentioned above, they used a variety of perfusates and 12 hours of OCS perfusion prior to transplant. The perfusate was an important factor in appropriate reconditioning of the organ. In these experiments, the OCS system was actually used as a static system. There may be situations where this is worthwhile. For instance, in extended criteria, organs that have a high rate of turn down, it may be too resource intensive to transport the device and personnel to the site. Instead, the same portable system could be used at the implant center once the organ arrived after a brief few hours of cold storage. This should only be utilized in select scenarios, as the real value of portable EVLP comes in the minimization of cold ischemic injury. The pilot study from Spratt and colleagues also utilized the OCS system in a static manner with reasonable success (22). Mohite and colleagues reported a case using the OCS system in a static manner after cold ischemia with a positive outcome as well (26). In this regard, the OCS becomes a very flexible platform that could be used portably for standard donors to avoid cold ischemic insults and also as a static system in situations that are more questionable and require longer preservation intervals.

Donor lung expansion over the next decade will be feasible with the use of EVLP. However, there needs to be a high throughput emphasis on a variety of interventions that may influence the recovery of the oxygenating alveolar units on EVLP prior to transplant. For instance, gene therapy, stem cell therapy, post-conditioning agents, and altered ventilator, flow or temperature settings are all possible interventions that could lead to improved results after preservation of extended criteria organs.

\section{Conclusions}

Portable normothermic EVLP with the OCS Lung is a major technological advancement in lung transplantation. There is a growing body of evidence that will allow us to identify the ideal scenarios for its use. Currently, it is indicated in the United States for standard donor lung preservation and is being evaluated in ECD. Future research is needed to evaluate ways to improve outcomes even further in ECD preserved with machine perfusion, an ideal platform for therapeutic interventions.

\section{Acknowledgments}

We thank Ronnetta Eaton for manuscript review.

\section{Footnote}

Conflicts of Interest: Dr. Loor receives travel support for conferences related to the clinical evaluation of the Organ Care System by Transmedics. Dr. Loor's institution, Baylor College of Medicine, receives grant support related to his involvement in the OCS clinical research studies. The other authors have no conflicts of interest to declare.

\section{References}

1. Heron M. Deaths: Leading Causes for 2016. Natl Vital Stat Rep 2018;67:1-77.

2. Chambers DC, Cherikh WS, Goldfarb SB, et al. The International Thoracic Organ Transplant Registry of the International Society for Heart and Lung Transplantation: Thirty-fifth adult lung and heart-lung transplant report-2018; Focus theme: Multiorgan Transplantation. J Heart Lung Transplant 2018;37:1169-83.

3. Valapour M, Skeans MA, Smith JM, et al. OPTN/SRTR 2015 Annual Data Report: Lung. Am J Transplant 2017;17 Suppl 1:357-424.

4. Whitson BA, Prekker ME, Herrington CS, et al. Primary graft dysfunction and long-term pulmonary function after lung transplantation. J Heart Lung Transplant 2007;26:1004-11.

5. Diamond JM, Arcasoy S, Kennedy CC, et al. Report of the 
International Society for Heart and Lung Transplantation Working Group on Primary Lung Graft Dysfunction, part II: Epidemiology, risk factors, and outcomes-A 2016 Consensus Group statement of the International Society for Heart and Lung Transplantation. J Heart Lung Transplant 2017;36:1104-13.

6. Loor G. EVLP: Ready for Prime Time? Semin Thorac Cardiovasc Surg 2019;31:1-6.

7. Warnecke G, Moradiellos J, Tudorache I, et al. Normothermic perfusion of donor lungs for preservation and assessment with the Organ Care System Lung before bilateral transplantation: a pilot study of 12 patients. Lancet 2012;380:1851-8.

8. Cypel M, Yeung JC, Liu M, et al. Normothermic ex vivo lung perfusion in clinical lung transplantation. $\mathrm{N}$ Engl J Med 2011;364:1431-40.

9. Cypel M, Yeung JC, Machuca T, et al. Experience with the first 50 ex vivo lung perfusions in clinical transplantation. J Thorac Cardiovasc Surg 2012;144:1200-6.

10. Warnecke G, Van Raemdonck D, Smith MA, et al. Normothermic ex-vivo preservation with the portable Organ Care System Lung device for bilateral lung transplantation (INSPIRE): a randomised, open-label, non-inferiority, phase 3 study. Lancet Respir Med 2018;6:357-67.

11. Diamond JM, Lee JC, Kawut SM, et al. Clinical risk factors for primary graft dysfunction after lung transplantation. Am J Respir Crit Care Med 2013;187:527-34.

12. Snell GI, Yusen RD, Weill D, et al. Report of the ISHLT Working Group on Primary Lung Graft Dysfunction, part I: Definition and grading-A 2016 Consensus Group statement of the International Society for Heart and Lung Transplantation. J Heart Lung Transplant 2017;36:1097-103.

13. Loor G WG, Villavicencio M, Smith M, Kukreja J, Ardehali A, Hartwig M, Daneshmand M, Hertz M, Haverich A, Madsen J, and Van Raemdonck, D. . Results of the OCS Lung EXPAND International Trial Using Portable Normothermic OCS Lung Perfusion System (OCS) to Recruit and Evaluate Extended Criteria Donor (ECD) Lungs. J Heart Lung Transplant 2018;37:S147.

14. Fisher A, Andreasson A, Chrysos A, et al. An observational study of Donor Ex Vivo Lung Perfusion in UK lung transplantation: DEVELOP-UK. Health Technol Assess 2016;20:1-276.

15. Whitson BA, Shukrallah B, Mulligan MS, et al. Ex-Vivo Lung Perfusion in Donation After Circulatory Death Lung Transplantation Increases Donor Utilization: Analysis of the NOVEL Extension Trial. J Heart Lung Transplant
2018;37:S147-8.

16. Sanchez PG, Davis RD, D'Ovidio F, et al. The NOVEL Lung Trial One-Year Outcomes. J Heart Lung Transplant 2014;33:S71-2.

17. Zeriouh M, Sabashnikov A, Mohite PN, et al. Utilization of the organ care system for bilateral lung transplantation: preliminary results of a comparative study. Interact Cardiovasc Thorac Surg 2016;23:351-7.

18. Schiavon M, Faggi G, Rebusso A, et al. Extended criteria donor lung reconditioning with the organ care system lung: a single institution experience. Transpl Int 2019;32:131-40.

19. Loor G, Howard BT, Spratt JR, et al. Prolonged EVLP Using OCS Lung: Cellular and Acellular Perfusates. Transplantation 2017;101:2303-11.

20. Spratt JR, Mattison LM, Iaizzo PA, et al. The ABCs of autologous blood collection for ex vivo organ preservation. J Thorac Cardiovasc Surg 2018;155:433-5.

21. Sommer W, Salman J, Avsar M, et al. Prediction of transplant outcome after 24-hour ex vivo lung perfusion using the Organ Care System in a porcine lung transplantation model. Am J Transplant 2019;19:345-55.

22. Spratt JR, Mattison LM, Kerns NK, et al. Prolonged Preservation and Evaluation of Human Lungs With Portable Normothermic EVLP. J Heart Lung Transplant 2018;37:S242.

23. Mohite PN, Sabashnikov A, Garcia Saez D, et al. Utilization of the Organ Care System Lung for the assessment of lungs from a donor after cardiac death (DCD) before bilateral transplantation. Perfusion 2015;30:427-30.

24. Spratt JR, Mattison LM, Iaizzo PA, et al. An experimental study of the recovery of injured porcine lungs with prolonged normothermic cellular ex vivo lung perfusion following donation after circulatory death. Transpl Int 2017;30:932-44.

25. Spratt JR, Mattison LM, Iaizzo PA, et al. Lung transplant after prolonged ex vivo lung perfusion: predictors of allograft function in swine. Transpl Int 2018;31:1405-17.

26. Mohite PN, Maunz O, Popov AF, et al. Utilization of the organ care system as ex-vivo lung perfusion after cold storage transportation. Perfusion 2015;30:698-700.

Cite this article as: Lightle W, Daoud D, Loor G. Breathing lung transplantation with the Organ Care System (OCS) Lung: lessons learned and future implications. J Thorac Dis 2019;11(Suppl 14):S1755-S1760. doi: 10.21037/jtd.2019.03.32 